\title{
PCI IN OCTO AND NANOGENERIANS
}

\author{
Maddury Jyotsna
}

With increasing aging population in India and throughout the world we have to pay attention to treatment strategies for coronary artery disease patients who were above 80 years. Important and difficult concerns in this age group are presence of more extensive CAD with calcific lesions. Way back in 1992 itself there was a Scientific Session on the management of heart disease in the octogenarian by American College of Cardiology in Dallas [1]. According to Glen J. Kowalchuk et al the frequency of 0-, 1-, 2-, and 3-vessel and left the main CAD was 7, 14, 21, 57 and 13\%, respectively. Based on angiographic criteria, only 31\% had coronary anatomy amenable to PTCA with a clinical success rate of $83 \%$ [2].

Other challenges with Octogenarians are significantly higher rates of co-morbidities like hypertension, CAD, peripheral vascular disease, atrial fibrillation $(\mathrm{AF})$, chronic kidney disease, cerebrovascular disease, chronic obstructive pulmonary disease (COPD), arthritis, dyslipidemia, diabetes, and cardiomyopathy. They also have much higher rates of dementia, lower tolerances to sedation, and high risks for procedural and postprocedural complications. Bleeding risks are a major concern for octogenarians, and procedural anticoagulation and long-term antiplatelet therapy need to be carefully managed [3].

In this issue, an article by Anil et al on "Acute Results Of PCI For CAD In Octo And Nonagenarian Patients", discussed the difficulties and acute results following the $\mathrm{PCI}$ in 174 octo and Nonagenarian CAD population with max age of 99 years. In this study multivessel disease in not frequent. $69 \%$ had single vessel disease. This is mainly analysis done in patients already referred for $\mathrm{PCI}$, but on the angiographic analysis of all octo and Nonagenarian. As expected $88 \%$ of lesions required Predilatation due to the presence of tight narrowing or calcification.

Surprisingly procedural success was seen in 95.4\% patients which are comparable to younger age group

Article received on 01 JAN 2017, published on 31JAN 2017

Maddury Jyotsna ${ }^{1}$

'Professor \& HOU-IV, Department of Cardiology, NIMS, India.

Corresponding Author: Maddury Jyotsna

Email: mail2jyotsna@rediffmail.com patients and only $4 \%$ patients had procedural complications. These statistics are very favorable to do PCI in this age group then when compared to previous studies [2,4]. This may be due to improvement in stent technology with overall PCI technology improvement. But we have to accept this improvement of acute PCI success with pin of salt as there is no detail description about the complexity of the lesion and/or patient.

Any why, this article gives us hope of using PCI as primary modality of management in octo and Nonagenarian, in contrast to previous studies concentrating on CABG $[5,6]$.

\section{REFERENCES :}

1. Mortimer J. Buckley, Richard J. Gray, Geoffrey 0. Hartzler, and Sylvan Lee Weinberg. Management of heart disease in the octogenarian. Clin. Cardiol. 15,479481 (1992).

2. Glen J. Kowalchuk, MD, Samuel C. Siu, MD, Stanley M. Lewis, MD. Coronary artery disease in the octogenarian: Angiographic spectrum and suitability for revascularization December 1, 1990: Volume 66, Issue 19, Pages 1319-1323.

3. Richard J. Merschen, EdS, RT(R)(CV), RCIS, Ibrahim Khormi, MS, RT(R)(T)(CT)(MR), Lamis Jada, MS, RT(MR), Tamara Kobakhidze, MS, RT(R), Jennifer Robinson, MS, RT(R). Octogenarians and Issues in Cath Lab Care. Cath Lab Digest Volume 22 - Issue 9 September 2014.

4. Cathy Johnman; Keith G Oldroyd; Jill P Pell. Elective Percutaneous Coronary Intervention in the Elderly Patient. Aging Health. 2011;7(2):271-281.

5. Abdulhameed Aziz, Anson M. Lee, Michael K. Pasque, Jennifer S. Lawton, Nader Moazami, Ralph J. Damiano, Marc R. Moon. Evaluation of Revascularization Subtypes in Octogenarians Undergoing Coronary Artery Bypass Grafting. Circulation. 2009;120:S65-S69.

6. Ko W1, Gold JP, Lazzaro R, Zelano JA, Lang S, Isom OW, Krieger KH.Survival analysis of octogenarian patients with coronary artery disease managed by elective coronary artery bypass surgery versus conventional medical treatment. Circulation. 1992 Nov;86(5 Suppl):II191-7. 